\title{
Data collection from road traffic accidents
}

\author{
A. F. DOVE ${ }^{1}$, J. C. G. PEARSON ${ }^{2}$ AND P. A. M. WESTON ${ }^{1}$ \\ Departments of ${ }^{1}$ Accident and Emergency Medicine and ${ }^{2}$ Community Health, University \\ Hospital, Nottingham, England
}

\section{SUMMARY}

A method of improving information about injuries sustained in road traffic accidents is described. It was achieved by combining data from the police with that from the accident and emergency department in such a way that patient confidentiality was preserved. This improved data base shows that present estimates of the number of injuries sustained on the road are too low and that assessments of their severity are probably inaccurate.

\section{INTRODUCTION}

Planning at national and local levels to deal with the consequences of injuries sustained in road traffic accidents is dependent on data obtained from police reports. At present, statistics about both the number of people injured and the severity of their injuries are gathered by the local authority traffic investigation unit from reports made by the police.

This study was commissioned by the road accident analysis group of Odense University acting as the project coordinator for the World Health Organization. Similar exercises were undertaken in Sweden, Poland, Morocco, India and Denmark. The studies were aimed at investigating methods of collecting comparable data about injuries following road traffic accidents from a number of different countries. In particular, the study was designed to explore methods of linkage of hospital and police data whilst preserving patient confidentiality.

In addition to W.H.O. objectives, we also defined local aims for Nottingham. These included an assessment of the number of accidents not reported to the police and the 
validity of the police assessment of injury. We also used this study to explore the likely $\frac{\mathbb{D}}{0}$ value of hospital inpatient statistics as indicators of the effects of injury, and to see 3 whether an assessment of the likely length of stay could be made when the patient was? first seen.

Although our findings are directly applicable only to Nottingham, we have no reason to suspect that they would not be more widely relevant.

\section{MATERIAL AND METHODS}

For a period of 3 months, information was collected about every road traffic accident $\bar{\omega}$ victim who attended the Accident and Emergency Department at the University $\frac{\Phi}{3}$ Hospital, Nottingham. The hospital is the only major accident centre for a population of about 804000 . The area served includes heavy and light industry, agriculture, a stretch $\omega$ of motorway and several major trunk roads. All severely injured patients within this $\vec{\oplus}$ area attend the University Hospital. Any patient who required continuing hospital care을 would have been referred to the Accident and Emergency Department. The area covered by this hospital overlaps areas covered by other accident departments at $\mathbb{\mathbb { D }}$ Leicester, Derby and Mansfield. There are also two smaller hospitals (Newark and $\underset{\mathbb{D}}{\stackrel{\circ}{0}}$ Grantham) from which all seriously ill patients are transferred to Nottingham. Most $\frac{}{8}$ patients who die in road traffic accidents are brought to hospital for certification of $\frac{\mathbb{Q}}{-}$ death. During the period of this study, arrangements were made for us to be notified ô $\overrightarrow{0}$ all deaths by the Coroner's Office.

During the study period, the following protocol was followed:

(1) In accordance with our usual practice, every patient who attended the Accident and Emergency Department had the following details recorded: name, address, age, sex, marital status, source of injury, method of referral, time, date and day of $\frac{\mathrm{Q}}{\mathscr{\mathrm { Q }}}$ arrival.

(2) In addition to this basic data, during the period of the study, a rubber stamp was $\frac{\bar{\circ}}{3}$ applied to the Accident and Emergency records of all road traffic accident victims. This reminded the doctor who treated them to record details of the accident and the patient's situation in it.

(3) The Accident and Emergency record was completed with a clinical diagnosis and disposal.

(4) The following day the record was photocopied and the data transferred on to a special form by a clerk who was employed for the purpose. At this stage, the information was coded according to an agreed protocol for later analysis.

(5) The partly completed survey form and record sheet passed to one of three senior $\stackrel{D}{0}$ doctors who recorded the Abbreviated Injury Scale score (AIS). From the AIS score, the Injury Severity Score (ISS) was calculated.

(6) For those patients who had been admitted to hospital, the senior doctor also 0 estimated the length of admission. The assessment was whether the stay would be short (less than 3 nights), medium (4 to 28 nights) or long (more than 28 nights).

(7) Actual length of admission was subsequently extracted from the hospital notes and $\stackrel{0}{\odot}$ encoded in the same way as the estimated length of stay. 
(8) Clinical information about patients who were certified dead on arrival at hospital or who died after admission was obtained from the post-mortem report.

(9) Linkage with police data was achieved by the hospital road traffic accident clerk. Because each patient involved in a traffic accident is charged a fee, part of the police report form is sent to the hospital. This form carries the name of those known to have been involved in the accident. For the duration of this study, it also carried the police accident number. This enabled data to be obtained from the main part of the police accident form which contains information about each injured individual. The information required was extracted by the road accident investigation team, a local authority body. Thus the flow of information was always from the police to the hospital. No hospital record or other information was relayed to the police.

(10) Completed forms were passed to the Department of Community Health in the University of Nottingham for computer analysis.

In the early stages of the survey, there was confusion about some phrases in the coding protocol: these were quickly resolved and did not affect the data.

Coding of the injury score was taken from the Abbreviated Injury Score of the American Automotive Association (1980): the ISS was calculated by adding the squares of the highest AIS scores achieved in the three worst affected body areas. For diagnostic coding, the World Health Organization Classification of Disease (1977) was used.

The police form is completed by the reporting police officer. In addition to the names of people injured, he recorded an assessment of the severity of the injury. The categories used are: (1) Dead; (2) Severe Injury; and (3) Slight Injury.

For the purposes of this study, we divided patients into five groups according to their injury severity score. The categories were: ISS: 0; ISS: 1-3; ISS: 4-9; ISS: 10 and over; died. Police category 2 was, therefore, subdivided into moderate injury (ISS: 4-9) and serious injury (ISS: $10+$ ).

\section{RESULTS}

During the period of the study, a total of 715 patients attended the Accident and Emergency Department following a road traffic accident. Fifty patients in the study had accidents outside the Nottingham Area and were excluded from the analysis of results. Conversely, information from the police suggests that a number of patients were treated in adjacent hospitals following accidents in Nottingham. Because the flow of information was always from the police to us, this error was not quantifiable. At least 70 patients sought treatment only from their general practitioner.

For 247 of the 715 patients (34.5\%), no linked police record was forthcoming: this group of patients of whom the police were unaware was examined in greater detail. Distribution as regards age and sex was the same as those for whom a linked record was obtained. A high number of accidents to pedal cyclists $(63 \%)$ were not reported (Table 1). There was a significant degree of injury within the group of patients who were not known to the police (Table 2). 
Table 1 Numbers of patients for whom linked records were received'

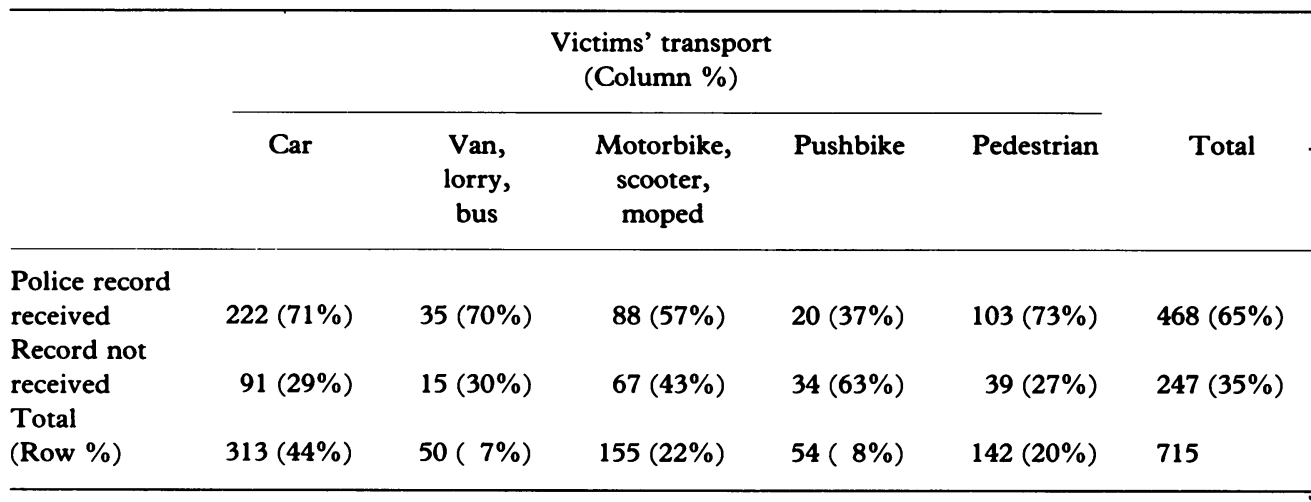

'One patient's transport was not recorded.

Table 2 Severity of injury and police record

\begin{tabular}{|c|c|c|c|c|c|c|}
\hline & \multicolumn{5}{|c|}{ Injury severity score } & ת \\
\hline & 0 & $1-3$ & 4-9 & $10+$ & Died & $\frac{\mathrm{C}}{2}$ \\
\hline Police record received & 26 & 321 & 70 & 45 & 6 & $\underline{\mathbb{D}}$ \\
\hline No police record received & 23 & 166 & 37 & 20 & 1 & 융 \\
\hline
\end{tabular}

Linked records were found for 468 patients, the police severity was recorded for except one. The relationship between the police severity scale and the ISS is shown in Table 3.

Although the severe injuries were mostly admitted to hospital, $73(14 \%)$ of thos discharged had injuries which were likely to carry a significant morbidity (Table 4 ). $\overrightarrow{0}$

The senior doctor estimated the likely duration of hospital stay for the 134 patients who were admitted. These estimates agreed in $58 \%$. Of those for whom the estimates did not agree, $17 \%$ of estimates were shorter than the actual stay and $25 \%$ were longer:

Table 3 Police value and injury severity score

\begin{tabular}{|c|c|c|c|c|c|c|}
\hline \multirow[b]{2}{*}{ Police value } & \multirow[b]{2}{*}{0} & \multirow[b]{2}{*}{$1-3$} & \multicolumn{3}{|c|}{ Injury severity score } & \multirow[b]{2}{*}{ Tota } \\
\hline & & & 4-9 & $10+$ & Died & \\
\hline 3 (slight) & 21 & 275 & 15 & 6 & - & 317 \\
\hline 2 (serious) & 2 & 47 & 54 & 41 & - & 144 \\
\hline 1 (died) & - & - & - & - & 6 & 6 \\
\hline Total & 23 & 322 & 69 & 47 & 6 & 467 \\
\hline
\end{tabular}


Table 4 Severity of injury related to hospital admission

\begin{tabular}{lrrrrr}
\hline & \multicolumn{3}{c}{ Injury severity score } & \\
\cline { 2 - 5 } & 0 & $1-3$ & $4-9$ & $10+$ & Total \\
\hline Admitted & 1 & 32 & 40 & 61 & 134 \\
Discharged & 45 & 456 & 67 & 6 & 574 \\
Total & 46 & 488 & 107 & 67 & 708 \\
\hline
\end{tabular}

(Seven patients died)

\section{DISCUSSION}

At present, statistics derived from police reports are widely promulgated and used for planning at both national and local levels. This study has shown that such statistics give a low estimate of the number of accidents and an inaccurate picture of the severity of injury resulting from them. One consequence of this is that comparison of annual accident rates is difficult because it is impossible to decide whether an increase is real or only the result of improved reporting.

This study was undertaken to test a simple and effective way of obtaining more complete information about injuries resulting from Road Traffic Accidents. It was successful, no major problems were found in the system of linkage or in the coding by which we operated it. We believe that the resulting data provides more complete information than is available from any source at present.

Overall, nearly $35 \%$ of injuries were not reported to the police. This deficit did not appear to be due to clerical error. The degree of under-reporting that we found was slightly higher than previous reports (Bull et al., 1973; Hobbs et al., 1979) but our findings about the groups of patients who are not being notified to the police is similar. The greatest underestimate is of pushbike riders where only $37 \%$ of injuries were reported. The linked study found similarly low levels in Sweden (Kruse, personal communication). A knowledge of the relative reporting rates of the different modalities of transport enables appropriate weighting to be given to accident statistics.

For minor injuries, the police severity value corresponded well with the Injury Severity Score: $93 \%$ of those classified as slightly injured by the police had an ISS of 3 or less. The Police category of 'severe', however, covered a wider range, with about one third having an ISS of 3 or less and about one third having an ISS of 10 or over, two patients who were classified as seriously injured were, in fact, uninjured. This police category covers such a wide range of actual injury that information derived from the police assessment is suspect.

It has been suggested that the analysis of the length of hospital stay gives a useful picture of morbidity resulting from accidents (Hobbs et al., 1979). We feel, however, that the picture is distorted. Few of those with no injury were admitted and most of those with serious injuries were admitted. In the group with an ISS of 1 to 9 there are 
many with significant injuries who are discharged from hospital. A number of such patients will be referred to hospital outpatients for further care and it may be that their inclusion would help to improve the sample. We were unsuccessful in predicting the length of stay in hospital. This may be partly due to the differences in practice between hospital specialists but the same difficulty arises with any system which seeks to use length of stay as a criterion for severity of injury.

In summary, we believe that the linked data collection system described herein, is a straightforward way to obtain relevant data about the number and severity of injuries $\stackrel{\mathbb{\Phi}}{\mathscr{\Phi}}$ sustained in road traffic accidents whilst preserving patient confidentiality.

\section{ACKNOWLEDGEMENT}

This work was supported by a grant from the Department of Health and Socialiw Security.

\section{REFERENCES}

The American Association for Automotive Medicine (1980) The Abbreviated Injury Scale. 1980 revision. The므 American Association for Automotive Medicine.

Bull J. P. \& Roberts B. J. (1973) Accident Analysis and Prevention 5, 45.

Hobbs C. A., Grattan E. \& Hobbs J. A. (1979) Classification of severity of injury by length of stay in hospita TRRL Laboratory Report No. 871.

World Health Organization (1977) Manual of the International Statistical Classification of Diseases, Injuries and Causes of Death. Geneva, W.H.O.

Received 25 Fune 1985; accepted 15 November 1985 